\title{
La liaison variable: des manuels scolaires japonais au jugement de locuteurs natifs
}

\author{
Samantha Ruvoletto $^{1}$ et Nori Kondo ${ }^{2}$ \\ ${ }^{1}$ Université de Lorraine, 23 Boulevard Albert 1er, 54000 Nancy \\ ${ }^{2}$ Nagoya University of Foreign Studies, 57, Takehoyama, Iwasakicho, Nisshin, Aichi, Japan
}

\begin{abstract}
Résumé. La liaison variable est un phénomène phonologique du français sujet à plusieurs contraintes para-linguistiques ou extra-linguistiques comme, par exemple, des facteurs sociolinguistiques et stylistiques (Durand et al., 2011). Sa variation fait que cette liaison pose des difficultés quand il s'agit de l'expliquer dans les manuels de français langue étrangère comme montré par Kondo (2012) dans son étude sur les manuels de FLE publiés au Japon. Dans cette étude, nous essayons de comprendre si les réalisations de la liaison variable présentes dans ces manuels correspondent aux réalisations potentielles de 30 locuteurs natifs (divisés en deux groupes: Groupe Formel et Informel). Dans une tâche de jugement, les participants entendent 50 phrases dans deux conditions: "liaison réalisée » (ex. il est $[t]$ originaire [ile.to.вi.zi.nєь]); «non réalisation liaison» (ex. il est originaire [ile.o.вi.zi.nєь]) et doivent décider s'ils prononceraient plutôt la première ou la deuxième phrase. En comparant nos résultats avec les réalisations des manuels, nous nous rendons compte que si nous analysons exclusivement les contextes syntaxiques, les manuels proposent une réalisation de la liaison qui est plus normative que variable. Cependant si nous nous concentrons sur une analyse «mot à mot ", ces mêmes manuels proposent des pourcentages de réalisation plus proches de l'usage qu'à la norme. Ces résultats nous permettent de réfléchir sur comment présenter la liaison variable dans les manuels de FLE.
\end{abstract}

\begin{abstract}
The variable liaison: from the French $L 2$ textbooks printed in Japan to native speakers' judgment. The variable liaison is a French optional phonological phenomenon depending on several sociolinguistic and stylistic parameters (Durand et al., 2011). Variable liaison acquisition seems to be a complicated task for L2 speakers. French L2 textbooks printed in Japan does not help this process showing some lacks in the way they presented this phenomenon (Kondo, 2012). In this study, we try to understand if the realizations of variable liaisons found in French L2 textbooks printed in Japan match native speakers' judgment. We tested 30 French native speakers by a judgement task: after hearing two sentences, one with liaison (e.g. il est[t]originaire [ile.to.вi.zi.nes]) and the other without (e.g.il est originaire [ile.o.ri.zi.nes]), they had to choose which one was more appropriate to the context (formal $v s$ informal). Comparing the
\end{abstract}

\footnotetext{
${ }^{1}$ Corresponding author : samantha.ruvoletto@email
} 
results of our task to L2 French textbooks, we concluded that if we analyze syntactic contexts, textbooks printed in Japan presented the variable liaison as a categorical phenomenon even if it was optional for native speakers. On the contrary, if we focused on the words entailing liaison processes, the percentage of textbook realizations were closer to French speakers' use. These results allowed us to discuss the best approach that had to be adopted to introduce variable liaison in French L2 textbooks.

\section{La liaison : un phénomène variable}

La liaison est un phénomène de sandhi externe très fréquent en français. Les éléments impliqués dans le processus de liaison sont deux mots (Mot1 et Mot2) et une consonne de liaison (CL). Comme exemplifié en (1), une consonne latente, dite consonne de liaison (CL) apparaît en surface (le [z] dans [le.za.mi] vs les [le] en isolation où le $[\mathrm{z}]$ n'apparaît pas en surface).

\section{(1) Mot1 : les [le] + Mot2 : amis [ami] $\rightarrow$ les[z]amis [le.za.mi]}

La cohésion syntaxique entre Mot1 et Mot2 est posée comme déterminante pour la réalisation de la liaison : sans cohésion syntaxique, la liaison ne se fera pas. Les études des catégories syntaxiques possibles de Mot1 et Mot2 ont permis une première classification de la liaison faite par Delattre (1947) qui distingue trois catégories de liaison : obligatoire, facultative et interdite (résumées dans la Figure 1).

\begin{tabular}{|c|c|c|c|}
\hline & Liaisons obligatoires & Liaisons facultatives & Liaisons interdites \\
\hline Noms & $\begin{array}{l}\text { - Det + N /Adj } \\
\text { Un [n] ami } \\
\text { Deux [z] amis } \\
\text { - Adj + N } \\
\text { Un grand [t] ami }\end{array}$ & $\begin{array}{l}\text { - } \mathrm{N}_{\mathrm{pl}}+\mathrm{X} \\
\text { Les jeunes filles [z]? en } \\
\text { fleur } \\
\text { Les filles [z]? émues } \\
\text { Les jardins [z]? ont fleuri }\end{array}$ & $\begin{array}{l}-\mathrm{N}_{\mathrm{co}}+\mathrm{X} \\
\text { Le soldat// en fête } \\
\text { Le soldat// ému } \\
\text { Le soldat// est revenu }\end{array}$ \\
\hline Verbes & $\begin{array}{l}\text { - } \text { Proport }_{\text {Pre }}+\text { Vbe } \\
\text { lls }[z] \text { ont } \\
\text { - Vbe + Pro } \\
\text { Ont-[t] ils }\end{array}$ & $\begin{array}{l}\text { - Aux }+ \text { Vbe } \\
\text { Ils ont [t]? été } \\
\text { Je vais [z]? essayer } \\
\text { - Vbe }+X \\
\text { II arrivait [t]? à l'heure }\end{array}$ & \\
\hline $\begin{array}{l}\text { Mots } \\
\text { inva riables }\end{array}$ & $\begin{array}{l}\text { - Monosyll + X } \\
\text { E n [n] avion } \\
\text { Très [z] en retard } \\
\text { Trop [p] important }\end{array}$ & $\begin{array}{l}\text { Polysyll }+\mathbf{X} \\
\text { Pendant }[t] \text { ? une heure } \\
\text { Toujours }[z] \text { ? en retard } \\
\text { Extrêmement } \\
\text { important }\end{array}$ & $\begin{array}{l}\mathrm{Et}+\mathrm{X} \\
\mathrm{Et} / / \text { alors } \\
\mathrm{Et} / / \text { avec lui }\end{array}$ \\
\hline $\begin{array}{l}\text { Constructions } \\
\text { figées } \\
\text { et cas } \\
\text { particuliers }\end{array}$ & $\begin{array}{l}\text { Tout-[t]-à-coup } \\
\text { Les E tats }-[z] \text { Un is }\end{array}$ & & $\begin{array}{l}\text { H } \alpha \text { aspiré } n \\
\text { Des // héros } \\
\text { En } / / \text { haut } \\
\text { Cent } / / \text { huitième fo is }\end{array}$ \\
\hline
\end{tabular}

Figure 1. Classification de la liaison et exemples de Wauquier (2010 : 5) après Delattre (1947)

Dans cet article, nous nous concentrons sur le phénomène de la liaison facultative. Cela implique que le locuteur peut prononcer la séquence de deux mots soit avec liaison, soit sans liaison. La production de la liaison n'est pas fautive, et en même temps la nonproduction n'est pas une erreur. Comme nous pouvons le voir dans la Figure 1, dans le contexte nominal, nous trouvons des liaisons facultatives entre nom pluriel et Mot2 à voyelle initiale (ex. les filles[z]émues). Pour les contextes verbaux, entre auxiliaire et verbe (Ils ont[t]été) ou entre verbe et Mot2 commençant par une voyelle (Il arrivait[t]à l'heure). 
Pour les mots invariables, il y a la possibilité de liaison entre un mot polysyllabique et un nom à voyelle initiale (Pendant[t]une heure).

Grâce à l'analyse du corpus du projet Phonologie du Français Contemporain (PFC) et à son protocole d'enquête ${ }^{\mathrm{i}}$, les appellations de liaison comme «obligatoire » ou « facultative » ont été remises en question. Les analyses du corpus PFC (Mallet, 2008) montrent que la liaison est presque toujours réalisée dans les contextes suivants: déterminant + nom (les[z]amis); proclitique + verbe (vous[z]allez), verbe + enclitique (allez-[z]y); expressions figées (de temps[z]en temps). En revanche, la liaison est très variable dans le contexte monosyllabique $+X$. Dans ce contexte, les pourcentages de réalisation changent selon le type de mots monosyllabiques : la liaison est presque toujours réalisée avec en (13 non-réalisations pour 2006 réalisations), beaucoup moins réalisée après dans (59 non-réalisations pour 859 réalisations) et encore plus variable pour chez (18 nonréalisations pour 74 réalisations). Les résultats sur le contexte monosyllabique $+\mathrm{X}$ mettent en danger l'analyse de Delattre et sa classification, car, pour lui, ce dernier contexte devait être traité comme obligatoire. De plus, Durand \& Lyche (2008) remarquent que dans la catégorie définie par Delattre comme «obligatoire », il y a une variation de réalisation dépendant des phénomènes morphologiques et catégoriels. Ils proposent donc une nouvelle classification de la liaison fondée sur l'usage qui prévoit deux types de liaison : une liaison catégorique, toujours réalisée par les locuteurs ; une liaison variable qui est sujette à une variation de réalisations selon différentes composantes.

De plus, ils suggèrent d'adopter une description de la liaison en « mot à mot » qui donne plus d'attention à la production des locuteurs et aux contraintes para-linguistiques ou extra-linguistiques comme, par exemple, des facteurs sociolinguistiques et stylistiques qui sembleraient influencer sa réalisation et que nous détaillerons tout de suite.

\subsection{Les facteurs stylistiques, individuels et géographiques qui participent à la variation}

Pour les facteurs stylistiques, Delattre (1966) note une forte différence dans la fréquence des liaisons variables selon les registres utilisés par les locuteurs. Entre le registre familier et le registre plus soutenu, la fréquence de production de liaisons variables augmente. Ainsi, dans une conversation familière, il est plus fréquent de produire la liaison seulement dans les contextes obligatoires (des[z]hommes /illustres /ont /attendu). Dans une conversation soignée, le locuteur ajoute aux liaisons obligatoires les liaisons facultatives verbe-participe (des[z]hommes /illustres /ont $[\mathrm{t}]$ attendu). Pendant une conférence, plus de liaisons facultatives seront produites (des[z]hommes[z]illustres /ont $[\mathrm{t}]$ attendu) et enfin pour la récitation de vers, toutes les liaisons possibles seront réalisées (des[z]hommes[z]illustres[z]ont $[\mathrm{t}]$ attendu). Les trois modalités prévues dans le protocole $P F C$ permettent également d'avoir des données sur des «styles » différents, plus ou moins surveillés. Les résultats de l'analyse du corpus (Eychenne et al., 2014) montrent qu'un pourcentage plus élevé de liaison est produit en lecture (cela peut être influencé par la disponibilité de la forme orthographique, comme dans Laks, 2005). Le pourcentage de liaison est moins élevé en conversation guidée, et encore inférieur en conversation spontanée. Ces résultats confirment l'analyse de Delattre et soulignent que dans le parlé spontané, comme dans le registre familier, les liaisons variables sont moins réalisées.

Concernant les facteurs individuels qui influencent la réalisation de la liaison variable, des études (Malécot, 1975 ; Ashby, 1981 ; Ranson, 2008) montrent que les locuteurs plus âgés réalisent plus de liaisons facultatives, surtout en conversation spontanée.

En ce qui concerne le sexe des locuteurs, les résultats sont controversés : les analyses de Malécot (1975) ou Booij et De Jong (1987) montrent un usage plus fréquent de la liaison chez les femmes; en revanche, Ashby (1981) trouve une réalisation plus fréquente chez les hommes. Les résultats de recherches plus récentes montrent cependant qu'entre les 
productions des deux sexes, il n'y a pas de différence statistiquement significative (Durand et al., 2011).

Pour la variation de la liaison selon le niveau d'études, l'analyse d'échantillonnage PFC par Mallet (2008) ne montre aucune différence dans l'usage de la liaison.

Pour les facteurs géographiques, si des différences existent pour l'espace francophone «français langue seconde» (Afrique), pour l'espace francophone «français langue première », les données $P F C$ ne montrent pas de véritables différences dans l'emploi de la liaison ii . Pour la «France hexagonale», la recherche de Coquillon et al. (2010) souligne qu'il n'y a pas de différence importante entre la réalisation de la liaison au nord et au sud de la France.

\section{Les difficultés d'apprentissage de la liaison variable et sa représentation dans les manuels de français au Japon}

Étant donné que la liaison variable peut être ou ne pas être réalisée dans chaque contexte syntaxique et qu'elle est sujette à plusieurs facteurs para- et extra-linguistiques, sa caractéristique principale est la variabilité. Si on part de l'idée que l'acquisition de la liaison se fonde sur des critères de fréquence (Bybee, 2005), la liaison variable est moins fréquemment réalisée que la liaison obligatoire car pour chaque contexte syntaxique deux réalisations sont possibles (avec ou sans liaison). Cette variabilité rend ce phénomène phonologique compliqué et par conséquent difficile à acquérir. Chez les enfants francophones elle est maîtrisée tardivement, à environ 9-10 ans ( $v s$ la liaison obligatoire acquise vers 5 ans, Basset, 2000). Chez les apprenants du français, elle est un des phénomènes complexes à acquérir (cf. Racine \& Detey, 2016). Cependant, pour bien maîtriser la langue et s'approcher au plus des productions des locuteurs natifs, les apprenants doivent comprendre et réussir à gérer sa variabilité.

Par ailleurs, il est également difficile, en tant qu'enseignant de FLE, d'expliquer cette variabilité, propre à ce type de liaison. Dans les manuels publiés au Japon, la liaison est très souvent présentée simplement comme la prononciation de la consonne finale devant un mot à initiale vocalique. De plus, seuls deux types de liaisons sont souvent expliquées : la liaison obligatoire et la liaison interdite. La liaison variable n'est donc pas suffisamment mentionnée. L'étude de Kondo (2012) analyse les CDs disponibles avec les manuels de FLE (publiés au Japon et en France) et elle remarque que la réalisation de liaisons variables dans ces manuels est assez éloignée de la conversation quotidienne des francophones natifs et qu'elle se rapproche plus de la conversation radiophonique enregistrée dans le corpus d'Ågren (1973). Elle conclut en soulignant le caractère formel des manuels et un niveau de prononciation qui porte à faire référence à un registre soutenu.

Cependant, les types de conversations que nous pouvons trouver dans les manuels de FLE sont assez variés. Nous avons bien sûr des conversations formelles (interviews, cours universitaires, radio, télé, ...) mais également des conversations plus informelles (dialogues entre amis, famille) et des conversations neutres (dialogues chez le boulanger, dans une boutique, ...). Le caractère normatif qui a été observé dans les manuels semble donc être en contradiction avec le contenu parfois informel des manuels. Nous pouvons penser que ces manuels sont censés représenter le français de référence. Si c'est le cas, alors les réalisations de liaison variable dans ces manuels doivent être représentatives de l'attitude normative des locuteurs natifs dans différentes situations de communication (formelles $v s$ informelles).

Il est intéressant alors de vérifier si le jugement sur les différentes liaisons variables chez des locuteurs natifs correspond à celle chez les rédacteurs de manuels (eux aussi, natifs francophones). De même, en analysant le phénomène de la liaison variable, nous pouvons poser une autre question : quelle « langue » peut être enseignée dans les manuels ? En suivant la conclusion tirée par l'étude de Kondo (2012), le taux de réalisation de liaisons 
variables dans les manuels est plus proche de la conversation radiophonique et donc du registre soutenu. La prononciation dans les manuels est-elle aussi formelle ou bien informelle?

\section{Questions de recherche}

Dans cette étude, nous essayons de vérifier si les réalisations de liaisons variables présentes dans les manuels utilisés au Japon pour apprendre le français correspondent aux réalisations mises en œuvre par des locuteurs natifs. Pour les manuels, nous utiliserons un corpus construit en 2019 par Kondo à partir de documents sonores de manuels (parties contenant des dialogues) numérisés en Microsoft Word. Ce corpus se base sur 12 manuels publiés au Japon (niveau A1-A2, 21451 mots). Pour tester les réalisations chez des locuteurs natifs, nous avons créé une tâche de jugement présentant les mêmes contextes de liaison que dans ces manuels. Nous avons testé des francophones monolingues au moyen d'un jugement de phrases qui porte sur deux registres : formel et informel.

La comparaison entre la fréquence et le type de réalisation de liaison variable présente dans le corpus et émergente dans la tâche de jugement permettra de comprendre quelle « langue » est représentée dans ces manuels. Si le français des manuels fait référence à un registre formel, nous nous attendons à autant, voire plus de liaisons variables réalisées comparé à ce qu'on peut trouver chez les locuteurs natifs dans une situation de communication formelle.

La tâche de jugement que nous avons utilisée n'est pas une tâche de production, mais plutôt une tâche qui évalue des compétences métalinguistiques. Nous pouvons penser que le jugement de nos locuteurs ne représente pas l'usage mais plutôt la norme. Pour mieux comprendre nos résultats, nous allons également comparer les données de la tâche de jugement avec les corpus existants qui ont permis de décrire l'usage de la liaison variable.

Nous présentons en détails la méthodologie de la tâche de jugement dans le paragraphe suivant.

\section{Méthode}

\subsection{Participants}

Nous avons décidé de tester 30 étudiants francophones natifs en troisième année de Sciences du Langage. Des données personnelles, linguistiques, sociolinguistiques et socioéconomiques ont été récoltées au moyen d'un questionnaire. L'analyse des questionnaires nous montre que tous nos participants partagent le même niveau d'études $(B A C+2$ en Sciences du Langage) et le même âge (âge moyen $=20$ ans). Nos participants sont toutes de femmes et vivent toutes dans la même région Grand Est. L'homogénéité de notre échantillon fait que les facteurs individuels et géographiques qui influencent la réalisation de la liaison variable ne peuvent pas influencer nos résultats. Les étudiantes ont été testées une par une dans une salle silencieuse pendant environ 20 minutes en novembre 2019.

\subsection{Procédure}

Dans cette tâche de jugement, la participante entend deux phrases présentées dans deux conditions : une condition appelée « liaison réalisée » où la liaison cible est produite (ex. il est [t]originaire [ile.to.вi.zi.nєь]) ; une condition appelée "non-réalisation liaison » où la liaison cible n'est pas réalisée (ex. il est originaire [ile.ə.ьі.zi.nعь]). Après avoir écouté les deux phrases dans les deux conditions, la participante doit décider si elle prononcera plutôt 
la première ou la deuxième phrase. Étant donné que la réalisation de la liaison variable varie selon le type de registre utilisé, nous avons donné des consignes précises concernant la situation de communication dans laquelle elle doit s'imaginer pendant le jugement. Nous avons demandé à la moitié des participantes (groupe formel (GF) = 15 étudiantes) d'écouter les phrases en s'imaginant dans un contexte formel (ex. entretien de travail); l'autre moitié (groupe informel $(\mathrm{GI})=15$ étudiantes) devait s'imaginer dans un contexte informel (ex. conversation entre amis). La tâche est précédée d'une période d'entraînement qui continue jusqu'à ce que l'adulte comprenne la procédure. La liste des contextes est donnée en annexe.

Pour construire la tâche, nous avons utilisé le logiciel OpenSesame (Mathôt et al., 2012). Chaque participante dispose d'un ordinateur portable et d'un casque. Au début de la tâche, nous avons décidé d'afficher sur l'écran les consignes (Annexe 1). Après avoir lu la consigne, la participante peut cliquer sur le bouton «ok» qui apparaît au centre de l'écran pour débuter l'essai. La tâche se compose de 90 essais divisés par une pause en 2 blocs de 45 essais. La structure de chaque essai est la suivante :

- Fixation

$\mathrm{Au}$ centre de l'écran apparaît un point. Les participantes sont invitées à le regarder. Le point reste affiché 500 millisecondes sur l'écran.

- Présentation de l'image ( $(\ll$ suivie par la présentation acoustique de deux phrases L'image ( $(r$ s'affiche à l'écran. Pendant qu'elle reste affichée, la participante entend les deux phrases dans les deux conditions. Entre la première et la deuxième phrase, nous avons inséré une pause d'une durée de $750 \mathrm{~ms}$. Après avoir écouté la deuxième phrase, la participante doit accomplir la tâche. L'image ( $(\bullet$ reste affichée sur l'écran jusqu'à ce que le sujet donne une réponse en appuyant sur un des deux boutons possibles sur le clavier de l'ordinateur.

- Réponse

La tâche prévoit deux réponses possibles: « $1 »$ ou « 2 ». Les participantes doivent appuyer sur la touche $q$ pour répondre « 1 » et $m$ pour répondre « 2 » sur le clavier azerty de l'ordinateur portable. Des étiquettes «1» ou « 2 »'une couleur neutre sont collées aux touches.

Le logiciel OpenSesame enregistre automatiquement les temps de réponses (TR) et les réponses (1 ou 2). Les TR sont calculés à partir de la fin de la deuxième phrase entendue par la participante jusqu'au moment de la réponse (donc dès que les participantes appuient sur une des deux touches). L'ordre de présentation des essais dans les blocs est randomisé par le logiciel pour éviter des processus de facilitations ou de stratégies. De plus, nous avons randomisé également l'ordre de présentation des conditions. Cela implique que la participante entend parfois comme première phrase celle dans la condition «liaison nonréalisée » suivi de la condition « avec liaison ». Dans d'autres cas, l'ordre est inversé et elle entend d'abord la phrase dans la condition « liaison non-réalisée » et ensuite la phrase dans la condition « avec liaison ».

\subsection{Répertoire}

Pour la création des phrases tests, nous avons utilisé les mêmes contextes de liaison variable présents dans les CDs des manuels d'apprentissage utilisés au Japon et en France. Si dans les phrases tirées de ces manuels deux contextes de liaison variable étaient présents, nous avons proposé ces deux contextes dans deux phrases différentes. Cette procédure a été nécessaire pour pouvoir avoir un seul contexte de liaison par phrase et garantir que la réponse de la participante soit liée à ce seul et unique contexte. Nous avons donc 50 phrases tests dans deux conditions ( liaison réalisée » et «non-réalisation liaison»). À ces 50 phrases tests, nous avons ajouté 40 phrases fillers et 3 phrases entraînements (Annexe 2). Les phrases ont été enregistrées par un locuteur natif dans une salle silencieuse. 


\section{Résultats}

Nous présentons dans le Tableau 1 les résultats de notre tâche de jugement pour les deux groupes (GF et GI) ainsi que les pourcentages de réalisation de la liaison variable dans le corpus de manuels japonais.

Tableau 1. Pourcentage de réalisation de la liaison variable dans le corpus des manuels japonais et choix de la phrase où la liaison est réalisée dans la tâche de jugement par les Groupe Informel (GI) et Groupe Formel (GF) pour les différents contextes

\begin{tabular}{|c|c|c|c|c|}
\hline & \multirow{2}{*}{$\begin{array}{c}\text { \% Corpus de } \\
\text { manuels JP }\end{array}$} & \multicolumn{3}{|c|}{ \% choix de la phrase liaison réalisée } \\
\cline { 3 - 5 } & & $\begin{array}{c}\text { GF+GI } \\
(\mathbf{n}=\mathbf{3 0})\end{array}$ & $\begin{array}{c}\text { GI } \\
(\mathbf{n}=\mathbf{1 5})\end{array}$ & $\begin{array}{c}\text { GF } \\
(\mathbf{n}=\mathbf{1 5})\end{array}$ \\
\hline $\begin{array}{c}\text { Adverbe monosyllabique + adjectif } \\
\text { (très[z]élégant) }\end{array}$ & 95,65 & 73,34 & 73,33 & 73,35 \\
\hline $\begin{array}{l}\text { Nom pluriel + adjectif pluriel } \\
\text { (pratiques[z]écologiques) }\end{array}$ & 42,86 & 66,67 & 63,30 & 70,00 \\
\hline $\begin{array}{l}\text { Préposition monosyllabique + NP } \\
\text { (dans[z]un) }\end{array}$ & 100 & 88,34 & 80,00 & 90,00 \\
\hline Verbe + X & 37,86 & 50,88 & 43,33 & 52,90 \\
\hline $\begin{array}{c}\text { E Etre +X } \\
(\text { il est[t]originaire) }\end{array}$ & 65,69 & 56,90 & 51,60 & 61,00 \\
\hline $\begin{array}{c}\text { Autres verbes + X } \\
\text { présentez[z]à) }\end{array}$ & 6,15 & 59,21 & 63,70 & 54,70 \\
\hline
\end{tabular}

Nos analyses statistiques nous permettent de remarquer qu'il n'y a pas de différence statistiquement significative entre les groupes formel (GF) et informel (GI) en ce qui concerne les scores de choix des phrases présentées dans la condition «liaison réalisée » pour les tests $(\mathrm{t}(14)=1,29, \mathrm{p}=0,21)$, ni pour les temps de réponses $(\mathrm{t}(14)=0,43, \mathrm{p}=0,67)$. Nous avons étudié les résultats en considérant les différents contextes de liaison variable après la dernière classification $P F C$ présentée dans l'introduction: Adverbe monosyllabique + adjectif (ex. très élégant), Nom pluriel + adjectif pluriel (ex. pratiques écologiques), Préposition monosyllabique $+\mathrm{NP}$ (ex. dans un), Verbes $+\mathrm{X}$ (ex. Il est originaire). Nous n'avons pas étudié les contextes Adverbe polysyllabique + adjectif ni Préposition polysyllabique + NP car la liaison n'est jamais réalisée dans le corpus de manuels. Pour tous les contextes, la différence des scores de choix de la phrase présentée dans la condition « liaison réalisée » dans GF et GI n'est pas statistiquement significative (Adverbe monosyllabique + adjectif $\mathrm{t}(14)=0,01, \mathrm{p}=.239$; Nom pluriel + adjectif pluriel $\mathrm{t}(14)=0,46, \mathrm{p}=.18$; Préposition monosyllabique $+\mathrm{NP} \mathrm{t}(14)=2,09, \mathrm{p}=.06$; Verbes $+\mathrm{X}$, $\mathrm{t}(14)=3.07, \mathrm{p}=.01)$. Cela signifie que la situation de communication (formelle $v s$ informelle) n'influence pas le choix de réalisation/non-réalisation de la liaison variable chez nos participantes et que ces résultats ne dépendent pas du contexte syntaxique.

Étant donnée l'absence de différences pour les deux groupes, nous avons d'abord décidé de comparer les résultats de toutes les participantes (GF et GI confondus) aux résultats du corpus des manuels. Nous avons également choisi de comparer les données du corpus avec les résultats du groupe formel $(\mathrm{GF})$, pour vérifier si les réalisations de manuels peuvent plutôt faire référence aux réalisations produites dans une situation formelle.

Nous comparons alors les taux de liaison dans les manuels avec les taux de choix de la phrase dans la condition « liaison réalisée » chez toutes nos participantes (GF+GI) et par contexte dans le GF. La différence est statistiquement significative pour les contextes : 
Adverbe monosyllabique + adjectif pour GF+GI $\left(X^{2}=18,93 \mathrm{p}<.001\right)$ et pour GF $\left(X^{2}=27,45\right.$ $\mathrm{p}<.001)$; Nom pluriel + adjectif pluriel pour GF+GI $\left(X^{2}=11,40 \mathrm{p}<.001\right)$, et pour GF $\left(X^{2}=14.21 \mathrm{p}<.001\right)$; Préposition monosyllabique $+\mathrm{NP}$ pour GF+GI $\left(X^{2}=12,17 \mathrm{p}<.001\right)$ et pour GF $\left(X^{2}=10,53 \mathrm{p}<.05\right)$. Cependant cette différence n'est pas significative pour le contexte Verbes $+\mathrm{X}($ ex. Il est originaire $)$ pour $\mathrm{GF}+\mathrm{GI}\left(X^{2}=3,57, \mathrm{p}=0,06\right)$ mais elle est significative pour GF $\left(X^{2}=4,56, \mathrm{p}<.05\right)$.

Comme on peut le voir dans le Tableau 1, pour les contextes Adverbe monosyllabique + adjectif et Préposition monosyllabique + NP, les manuels japonais proposent plus de réalisations que ce que nos locutrices jugent devoir être réalisé en français même dans une situation formelle. Dans le cadre du contexte Préposition monosyllabique $+\mathrm{NP}$, dans les manuels la réalisation est présentée comme obligatoire. Au contraire, chez nos locutrices, elle est variable étant donné que seulement $66,67 \%$ de nos participantes, même en situation formelle, choisissent la réalisation de la liaison dans ce contexte. Pour Nom pluriel + adjectif pluriel les résultats sont inversés. Les locuteurs choisissent plus fréquemment la phrase où la liaison est réalisée.

Étant donnés les résultats statistiques particuliers pour le contexte Verbe $+\mathrm{X}$, nous avons essayé de l'analyser plus en détail. Nous nous sommes concentrées sur différentes liaisons variables selon que le Mot1 est le verbe être (ex. il est[t]originaire) ou d'autres verbes (ex. présentez[z]une). Nous avons comparé les taux de liaisons variables réalisés dans les manuels avec les taux de choix de la phrase dans la condition "liaison réalisée " chez toutes nos participantes (GF+GI) et dans le GF. La différence n'est pas statistiquement significative pour le verbe être, ni pour GF+GI $\left(X^{2}=1,61 \mathrm{p}=.20\right)$ ni pour GF $\left(X^{2}=1,66\right.$ $\mathrm{p}=.19)$. Pour Autres verbes, elle est significative pour $\mathrm{GF}+\mathrm{GI}\left(X^{2}=63,11 \mathrm{p}<.001\right)$ et pour $\mathrm{GF}$ $\left(X^{2}=35,74 \mathrm{p}<.001\right)$. On peut donc constater (Tableau 1) que les manuels japonais semblent bien représenter la réalisation de la liaison variable en français quand Motl est le verbe être conjugué mais pas pour les autres verbes où nos locuteurs jugent que la liaison doit être plus réalisée.

Nous avons décidé d'analyser plus en détails les résultats dans les contextes « Être + $\mathrm{X}$ » en proposant les pourcentages de réalisations avec Mot1 est ou suis. Les taux sont présentés dans le Tableau 2. En général, dans la tâche de jugement, les participantes du GF choisissent de plus réaliser la liaison que le GI après est $\left(X^{2}=36,33 \mathrm{p}<.001\right)$ et suis $\left(X^{2}=10,14 \mathrm{p}<.001\right)$. Cependant, en comparant les taux dans la tâche de jugement avec les taux des manuels, on se rend vite compte que le taux de réalisation de la liaison variable se rapproche plus des résultats du Groupe Formel dans le cas de « est $+X$ » $\left(X^{2}=1,21 \mathrm{p}=.27\right)$ et des résultats du Groupe Informel dans le cas de la liaison « suis $+X »\left(X^{2}=0,36 \mathrm{p}=.54\right)$.

Tableau 2. Pourcentage de réalisation de la liaison variable dans le corpus des manuels japonais, dans les corpus oraux de Mallet (2008) et d'Ågren (1973) et choix de la phrase où la liaison est réalisée dans la tâche de jugement par les Groupe Informel (GI) et Groupe Formel (GF) pour les Mots1 est et suis.

\begin{tabular}{|c|c|c|c|}
\hline & \multicolumn{2}{|c|}{$\hat{E}$ tre } \\
\hline & & $E s t+X$ & Suis $+X$ \\
\hline \multicolumn{2}{|l|}{ \% réalisations dans manuels JP } & 82,05 & 48,84 \\
\hline \multirow{2}{*}{$\begin{array}{l}\% \text { choix de la phrase avec liaison } \\
\text { réalisée }\end{array}$} & GF $(n=15)$ & 75 & 77,8 \\
\hline & GI $(n=15)$ & 32,25 & 56,6 \\
\hline \multicolumn{2}{|c|}{ \% réalisations dans l'étude de Mallet (2008: $283-284)$} & 43,87 & 13,49 \\
\hline \multicolumn{2}{|c|}{ \% réalisations dans l'étude d'Ågren (1973) } & 97 & 47 \\
\hline
\end{tabular}


Si nous analysons les résultats dans le Tableau 2, il est assez intéressant de remarquer que les locuteurs du GI choisissent la réalisation de liaison plus souvent pour « suis $+\mathrm{X}$ » que pour " est $+X$ » $\left(X^{2}=7,26 \mathrm{p}<.05\right)$. Ces résultats sont assez étonnants car l'étude de Mallet (2008) qui décrit l'usage de la liaison variable montre qu'elle est beaucoup moins réalisée après « suis » $(13.49 \%)^{\mathrm{iii}}$ qu'après « est» $(43.87 \%)$. De même, pour les locuteurs du GF, nous ne remarquons pas de différence pour les résultats de jugement selon le type de Mot1 impliqué $\left(X^{2}=0,22 \mathrm{p}=.64\right)$. On pourrait penser que le GF porte son jugement en faisant référence à une langue qui est plus formelle. Nous comparons alors les résultats avec les réalisations enregistrées par Ågren (1973) dans son corpus en situation formelle basé sur l'analyse d'enregistrements d'émissions radiophoniques. Néanmoins, même dans l'étude d'Ågren (1973), la liaison après « suis » (47\%) est moins fréquente qu'après « est » $(97 \%)$. Les données concernant leurs choix ne sont pas en accord avec les données récoltées dans les corpus qui analysent l'usage. Nous pouvons alors dire que, dans cette tâche de jugement, certaines données comme celles-ci peuvent être influencées par l'idée de «norme individuelle » et que cette norme, que les locutrices appliquent ici, ne correspond pas à l'usage. On ne saurait malheureusement dire si ces locutrices produiraient spontanément la liaison à l'oral en suivant leur jugement dans la tâche (ex. plus de liaison avec Mot1 suis qu'avec est). Cependant, ces données nous permettent de dire que la réalisation de la liaison dans ces contextes ne choque pas les locuteurs natifs.

Par ailleurs, si on compare les résultats de Mallet (2008) pour les Mots1 suis et est avec les pourcentages des manuels au Japon, nous nous rendons compte que, dans les deux cas, nous avons plus de réalisations de liaisons avec Motl est qu'avec Mot1 suis, même si les pourcentages enregistrés dans les manuels sont largement supérieurs à ceux de Mallet (2008). Cela peut nous faire penser que pour ces contextes, les manuels sont plus proches de l'usage que de la norme.

\section{Conclusion}

Nous avons comparé les taux de réalisations de liaisons variables dans les manuels utilisés au Japon à ceux des locuteurs natifs testés au moyen d'une tâche de jugement. En utilisant les mêmes contextes syntaxiques de liaisons variables et les mêmes Mot1 + Mot2 que dans les manuels, nous avons pu nous rendre compte que certains contextes sont présentés comme obligatoires dans les manuels (liaisons toujours réalisées), tandis que pour nos participantes il s'agit de contextes variables. C'est le cas du contexte Adverbe monosyllabique + adjectif (moins officiel) ou Préposition monosyllabique + NP (dans un), presque obligatoires dans les manuels, mais variables chez nos locutrices natives, dans une situation de communication informelle comme dans une situation formelle. Dans les manuels, la liaison entre Nom pluriel + adjectif pluriel (pratiques écologiques) et entre Autres verbes $+\mathrm{X}$ (présentez à) est moins représentée que chez nos locutrices, qui estiment la faire plus souvent, dans des situations aussi bien formelles qu'informelles.

À partir de nos données, nous pouvons aussi essayer de comprendre si la langue française présentée dans les manuels fait plutôt référence à un registre formel ou informel en analysant la réalisation de la liaison. Nous pouvons constater que la liaison variable est parfois plus réalisée que ce que nos locutrices estiment faire dans une situation formelle. En d'autres occasions, on la trouve moins réalisée dans les manuels. Mais est-ce que la réalisation de la liaison variable ici présente correspond véritablement à l'usage ? Prenons le cas de la liaison dans les contextes Adverbe monosyllabique + adjectif (moins officiel) et Préposition monosyllabique + NP (dans un). Dans ces deux contextes, les manuels 
présentent la liaison comme presque obligatoire, elle est toutefois identifiée comme variable par nos locutrices. La réalisation proposée par les manuels semble se référer à la norme classique et à l'ancienne classification de Delattre qu'on a présentée dans la Figure 1 (section 1) où ces deux contextes apparaissent dans la colonne « liaison obligatoire ». Après l'analyse du corpus $P F C$, ces contextes sont désormais insérés dans la liste des contextes où la liaison est variable et nos données, comme on l'a vu, confirment cette classification fondée sur l'usage.

Cependant, les données ne sont pas les mêmes lorsque l'on décide d'analyser de plus près le contexte Verbe $+\mathrm{X}$ et en particulier en présence des deux Mots 1 suis et est. On se rend compte que le taux de réalisation de la liaison variable dans les manuels au Japon se rapproche plus des résultats des corpus fondés sur l'usage de Mallet (2008) et d'Ågren (1973). De plus, les résultats de nos locutrices des Groupes Formel et Informel s'éloignent des pourcentages enregistrés dans ces corpus, nous faisant penser à un jugement qui ne se fonde pas sur l'usage pour ces contextes.

Nous pouvons donc conclure qu'il est probable que les manuels de FLE à la base de notre corpus essaient de présenter une langue plus « normative » que « variable » quand il s'agit des catégories syntaxiques classiques utilisées pour la classification de la liaison et de ses réalisations. Pourtant, la situation change quand nous analysons les réalisations « mot à mot». Dans les cas de Mots1 spécifiques (comme est ou suis), les manuels tiennent plus compte de l'usage et des recherches faites à partir des corpus. D'une part, les locuteurs natifs qui enregistrent les documents sonores pour les manuels sont plus normatifs dans la présentation de certains contextes syntaxiques "classiques », en nous proposant la liaison variable comme un phénomène plus régulier que ce qu'il est vraiment; d'autre part, leurs réalisations après certains Mots 1 assez fréquents correspondent plutôt à l'usage et rendent compte de façon plus fidèle de la production orale des francophones.

Nos données concernant la tâche de jugement nous permettent également de dire qu'en général, chez nos locutrices, la différence de réalisations de la liaison variable n'est pas influencée par le registre ou la situation de communication (formelle $v s$ informelle) et que ces résultats ne dépendent pas du contexte syntaxique. Cela est en contradiction avec la littérature qui a montré une différence de réalisation de la liaison variable selon les registres adoptés par les locuteurs (Delattre, 1966 ; Eychenne et al., 2014). Cependant cette absence de différence disparaît si nous analysons en détails le contexte de liaison être $+\mathrm{X}$. Nous remarquons alors que les participantes du GF choisissent plus facilement la phrase où la liaison est réalisée comparé au GI, que ce soit avec Mot1 est ou avec Mot1 suis. Les résultats de cette analyse "mot à mot» montrent l'importance de ne pas se limiter à analyser les contextes syntaxiques de la liaison pour comprendre sa réalisation. Comme cela a été montré par Durand et al. (2011), la description de la liaison doit tenir compte du type de Mot1 et Mot2 impliqués dans la liaison. Une description « mot à mot » peut donc être plus favorable, non seulement dans la description en linguistique mais aussi dans les manuels de FLE. Cela permet aussi d'imaginer une piste pour l'enseignement où, plutôt que de parler de «contextes syntaxiques », il faudra parler de mots qui entraînent plus ou moins facilement le processus de liaison.

Cette étude est une étude préliminaire et présente certaines limites. Concernant la tâche de jugement, il s'agit d'une tâche métalinguistique qui ne permet pas toujours de savoir si les choix de nos participantes sont basés sur l'usage ou sur la norme (ou sur les deux en proportions différentes). De plus, les participantes sont toutes des étudiantes en Sciences du Langage, déjà sensibles aux concepts de variabilité linguistique, sociolinguistique et régionale. Nous pouvons imaginer trouver d'autres résultats en testant une population différente. Pour la création des phrases tests, nous nous sommes fondées sur les phrases proposées par les manuels de FLE. Par conséquent, notre répertoire se compose de phrases de longueurs différentes où la liaison occupe une position différente (début, fin ou milieu de phrase). Cette absence d'homogénéité peut avoir influencé nos résultats. Une 
autre tâche de jugement qui teste d'autres locuteurs au moyen d'un répertoire de phrases plus homogène pourrait être le sujet d'une recherche future.

\section{Références bibliographiques}

Ågren, J. (1973). Étude sur quelques liaisons facultatives dans le français de conversation radiophonique: fréquence et facteurs. Uppsala : Acta Universitatis Upsaliensis.

Ashby, W., J. (1981). French liaison as a sociolinguistic Phenomenon. In William W. Cressy and Donna Jo Napoli (ed.). Linguistic Symposium on romance languages 9. Washington D.C.: Georgetown University Press, 46-57.

Basset, B. (2000). La liaison à 3, 7 et 11 ans: description et acquisition. Unpublished Master dissertation. University of Grenoble 3.

Booij, G. \& De Jong, D. (1987). The domain of liaison: theories and data. Linguistics, 25, 1005-1025.

Bybee, J. (2005). La liaison : effets de fréquence et constructions. Langages, 158, 24-37.

Coquillon, A., Durand J., Lyche C. \& Eychenne J. (2010). French Liaison: from global results to local varieties. Colloque international PHONLEX, Université de Toulouse II-Le Mirail (8-10 septembre 2010).

Delattre, P. (1947). La liaison en français, tendances et classification. The French Review, 21 (2), $148-157$.

Durand, J., Laks, B., Calderone, B. \& Tchobanov, A. (2011). Que savons-nous de la liaison aujourd'hui ?. Langue Française, 169 (1), 103-126.

Durand, J. \& Lyche, C. (2008). French liaison in the light of corpus data. Journal of French Language Studies, 18 (1), 33-66.

Eychenne, J., Lyche, C., Durand, J. \& Coquillon, A. (2014). Quelles données pour la liaison aujourd'hui : la question des corpus, dans C. Soum, A. Coquillon \& J.-P. Chevrot (eds) La liaison : approches contemporaines, Bern: Peter Lang, 33-60.

Kondo, N. (2012). La liaison facultative dans l'interaction spontanée et les manuels de FLE. Flambeau, Revue annuelle de la section française, Université des Langues Etrangères de Tokyo, 38, $31-51$.

Kondo, N. (2019). Phonetic Norm in French L2 Textbooks and Its Effect on L2 Learners' Speech Competence - The Case of French Liaison (poster), New Sounds the 9th International Symposium on the Acquisition of Second Language Speech, le 31 août 2019, Tokyo.

Laks, B. (2005). La liaison et l'illusion. Langage, 158, 101-125.

Malécot, A. (1975). French liaison as a fonction of grammatical, phonetic and paralinguistic variables. Phonetica, 32,161-179.

Mallet, G. (2008). La liaison en français : descriptions et analyses dans le corpus PFC. Thèse de doctorat, Université Paris Ouest-Nanterre-La Défense. 
Mathôt, S., Schreij, D. \& Theeuwes, J. (2012). OpenSesame: An open-source, graphical experiment builder for the social sciences. Behavior Research Methods, 44(2), 314-324.

Racine, I. \& Detey, S. (2015). Corpus oraux, liaison et locuteurs non natifs : de la recherche en phonologie à l'enseignement du français langue étrangère. Bulletin vals-asla, 102, 1-25.

Ranson, D. (2008). La liaison variable dans un corpus du français méridional : L'importance relative de la fonction grammaticale, in J. Durand, B. Habert \& B. Laks (eds)(2008) Congrès mondial de linguistique française. Recueil des résumés et CD-ROM des actes. Paris: Institut de Linguistique Française et EDP Sciences, 1657-1671.

Wauquier S. (2010), Acquisition de la phonologie « du » français, des usages à la structure. In M. Barra (ed), Le(s) français: formaliser la variation, Langue française, 168 (4), 127-144.

\section{Annexe}

Annexe 1. Consignes utilisées pour GF (Consigne 1) et GI (Consigne 2) dans la tâche de jugement

Consigne 1. Vous allez entendre des paires de phrases. Imaginez être dans une situation FORMELLE (entretien de travail, présentation, etc). Choisissez la phrase que vous trouvez la mieux prononcée et/ou la mieux formulée parmi les deux. Si vous préférez la phrase que vous avez entendue en premier, cliquez sur la touche 1 du clavier. Si vous préférez la deuxième phrase, cliquez sur la touche 2. ATTENTION ! Vous ne pourrez écouter les phrases qu'UNE FOIS ! Un petit entraînement va précéder le test. Cliquez sur "ok" pour commencer l'entraînement.

Consigne 2. Vous allez entendre des paires de phrases. Imaginez être dans une situation INFORMELLE (conversation entre amis). Choisissez la phrase que vous trouvez la mieux prononcée et/ou la mieux formulée parmi les deux. Si vous préférez la phrase que vous avez entendue en premier, cliquez sur la touche $1 \mathrm{du}$ clavier. Si vous préférez la deuxième phrase, cliquez sur la touche 2. ATTENTION ! Vous ne pourrez écouter les phrases qu'UNE FOIS ! Un petit entraînement va précéder le test. Cliquez sur "ok" pour commencer l'entraînement.

Annexe 2. Répertoire des phrases utilisées dans la tâche de jugement

\begin{tabular}{|c|l|l|}
\hline $\begin{array}{c}\text { Type } \\
\text { phrase }\end{array}$ & \multicolumn{1}{|c|}{ Liaison oui } & \multicolumn{1}{c|}{ Liaison non } \\
\hline \multicolumn{3}{|c|}{ TRAINING } \\
\hline 1 & Il a dessiné un petit éléphant & Il a dessiné un petit éléphant \\
\hline 2 & Marie ne mange plus de bon bons & Marie mange plus de bon bons \\
\hline 3 & Peux-tu me prêter ton stylo? & Tu peux me prêter ton stylo? \\
\hline \multicolumn{3}{|c|}{ TEST } \\
\hline 1 & $\begin{array}{l}\text { Il y a quelques années, suite à un } \\
\text { accident, j'ai dû changer ma voiture. }\end{array}$ & $\begin{array}{l}\text { Il y a quelques années, suite à un } \\
\text { accident, j'ai dû changer ma voiture. }\end{array}$ \\
\hline 2 & $\begin{array}{l}\text { après un choc dans ma vie, j'ai voulu } \\
\text { changer ma façon d'être }\end{array}$ & $\begin{array}{l}\text { après un choc dans ma vie, j'ai voulu } \\
\text { changer ma façon d'être }\end{array}$ \\
\hline 3 & C'est moins officiel & C'est moins officiel \\
\hline 4 & C'était un homme intelligent & C'était un homme intelligent \\
\hline 5 & J'ai rencontré une femme très & J'ai rencontré une femme très élégante. \\
\hline
\end{tabular}




\begin{tabular}{|c|c|c|}
\hline & élégante. & \\
\hline 6 & $\begin{array}{l}\text { Et là, je m'adresse tout } \\
\text { particulièrement aux randonneurs }\end{array}$ & $\begin{array}{l}\text { Et là, je m'adresse tout } \\
\text { particulièrement aux randonneurs }\end{array}$ \\
\hline 7 & $\begin{array}{l}\mathrm{Ce} \text { sac est tout simplement } \\
\text { extraordinaire ! }\end{array}$ & $\begin{array}{l}\mathrm{Ce} \text { sac est } \begin{array}{l}\text { tout } \\
\text { extraordinaire ! }\end{array} \\
\end{array}$ \\
\hline 8 & $\begin{array}{l}\text { La yourte est pour moi une évidence } \\
\text { entre mes pratiques écologiques et } \\
\text { mon travail quotidien }\end{array}$ & $\begin{array}{l}\text { La yourte est pour moi une évidence } \\
\text { entre mes pratiques écologiques et } \\
\text { mon travail quotidien }\end{array}$ \\
\hline 9 & $\begin{array}{l}\text { C'est là où il y a eu les Jeux } \\
\text { olympiques d'hiver. }\end{array}$ & $\begin{array}{l}\begin{array}{l}\text { C'est là où il y a eu les Jeux } \\
\text { olympiques d'hiver. }\end{array} \\
\end{array}$ \\
\hline 10 & Je fais du judo dans un club & Je fais du judo dans un club \\
\hline 11 & Je suis originaire du Mali & Je suis originaire du Mali \\
\hline 12 & j'habite en France chez un ami. & j'habite en France chez un ami. \\
\hline 13 & $\begin{array}{l}\text { Pendant une semaine, nous testerons } \\
\text { des participants }\end{array}$ & $\begin{array}{l}\text { Pendant une semaine, nous testerons } \\
\text { des participants }\end{array}$ \\
\hline 14 & $\begin{array}{l}\text { Pendant les vacances, nous irons à } \\
\text { Domremy. }\end{array}$ & $\begin{array}{l}\text { Pendant les vacances, nous irons à } \\
\text { Domremy. }\end{array}$ \\
\hline 15 & Elle est à Paris pour le travail & Elle est à Paris pour le travail \\
\hline 16 & Elle habite à Nancy depuis un mois. & Elle habite à Nancy depuis un mois. \\
\hline 17 & $\begin{array}{l}\text { En plus, j'adore ma nièce, la fille de } \\
\text { mon frère, elle est adorable ! }\end{array}$ & $\begin{array}{l}\text { En plus, j'adore ma nièce, la fille de } \\
\text { mon frère, elle est adorable! }\end{array}$ \\
\hline 18 & $\begin{array}{l}\text { Mes enfants font leur premier } \\
\text { bonhomme de neige dans le jardin, Ils } \\
\text { rient, ils sont heureux! }\end{array}$ & $\begin{array}{l}\text { Mes enfants font leur premier } \\
\text { bonhomme de neige dans le jardin, Ils } \\
\text { rient, ils sont heureux! }\end{array}$ \\
\hline 19 & Je suis invitée au cinéma demain soir. & Je suis invitée au cinéma demain soir. \\
\hline 20 & Je vais chez eux demain soir & Je vais chez eux demain soir \\
\hline 21 & $\begin{array}{l}\text { Nous sommes ouverts tout l'après- } \\
\text { midi. }\end{array}$ & $\begin{array}{l}\text { Nous sommes ouverts tout l'après- } \\
\text { midi. }\end{array}$ \\
\hline 22 & $\begin{array}{l}\text { Nous sommes fermé de dix heures à } \\
19 \text { heures. }\end{array}$ & $\begin{array}{l}\text { Nous sommes fermé de dix heures à } 19 \\
\text { heures. }\end{array}$ \\
\hline 23 & C'est un peu cher mais c'est bien! & C'est un peu cher mais c'est bien! \\
\hline 24 & $\begin{array}{l}\text { Ça va, mais je suis un peu fatigué et } \\
\text { j'ai mal au dos. }\end{array}$ & $\begin{array}{l}\text { Ça va, mais je suis un peu fatigué et } \\
\text { j'ai mal au dos. }\end{array}$ \\
\hline 25 & J'aimerais aussi aller au cinéma & J'aimerais aussi aller au cinéma \\
\hline 26 & $\begin{array}{l}\text { J'aimerai qu'il soit intelligent, bien } \\
\text { sûr, mais pas toujours le nez dans les } \\
\text { livres }\end{array}$ & $\begin{array}{l}\text { J'aimerai qu'il soit intelligent, bien } \\
\text { sûr, mais pas toujours le nez dans les } \\
\text { livres }\end{array}$ \\
\hline 27 & Ah oui, mais c'est aussi très chic ! & Ah oui, mais c'est aussi très chic ! \\
\hline 28 & $\begin{array}{l}\text { Et je voudrais aussi le dernier roman } \\
\text { d'Amélie Nothomb }\end{array}$ & $\begin{array}{l}\text { Et je voudrais aussi le dernier roman } \\
\text { d'Amélie Nothomb }\end{array}$ \\
\hline 29 & Il faut qu'il y ait une terrasse & Il faut qu'il y ait une terrasse \\
\hline 30 & C'est une sauce blanche & C'est une sauce blanche \\
\hline 31 & Vous présentez une pièce d'identité & Vous présentez une pièce d'identité \\
\hline 32 & Je vais appeler Hélène & Je vais appeler Hélène \\
\hline 33 & $\begin{array}{l}\begin{array}{l}\text { Vous voulez être infirmier ou } \\
\text { photographe? }\end{array} \\
\end{array}$ & $\begin{array}{l}\begin{array}{l}\text { Vous voulez être } \\
\text { photographe? }\end{array} \\
\end{array}$ \\
\hline 34 & Je vais être en retard. & Je vais être en retard. \\
\hline 35 & $\begin{array}{l}\text { Elles me plaisent beaucoup et, en plus, } \\
\text { elles sont écolo! }\end{array}$ & $\begin{array}{l}\text { Elles me plaisent beaucoup et, en plus, } \\
\text { elles sont écolo! }\end{array}$ \\
\hline 36 & je suis autrichienne. Enchantée! & je suis autrichienne. Enchantée! \\
\hline 37 & Elle est anglaise & Elle est anglaise \\
\hline
\end{tabular}




\begin{tabular}{|c|c|c|}
\hline 38 & Je suis allé à Rouen & Je suis allé à Rouen \\
\hline 39 & Certains sont arrivés depuis des mois & Certains sont arrivés depuis des mois \\
\hline 40 & Nous sommes allés prendre un bain & Nous sommes allés prendre un bain \\
\hline 41 & Son copain vit à Bruxelles, & Son copain vit à Bruxelles, \\
\hline 42 & il travaille pour l'Union européenne & il travaille pour l'Union européenne \\
\hline 43 & $\begin{array}{l}\text { Je suis né aux États-Unis mais je vis à } \\
\text { Madagascar. }\end{array}$ & $\begin{array}{l}\text { Je suis né aux États-Unis mais je vis à } \\
\text { Madagascar. }\end{array}$ \\
\hline 44 & je suis à Bruxelles toute la journée & je suis à Bruxelles toute la journée \\
\hline 45 & Il est en face de la Fnac & Il est en face de la Fnac \\
\hline 46 & Ici, nous sommes au centre-ville & Ici, nous sommes au centre-ville \\
\hline 47 & On peut y manger des légumes & On peut y manger des légumes \\
\hline 48 & Il m'a proposé d'y aller ensemble & Il m'a proposé d'y aller ensemble \\
\hline 49 & Il est un peu timide & Il est un peu timide, \\
\hline 50 & $\begin{array}{l}\text { Il est studieux, mais il aime aussi } \\
\text { s'amuser. }\end{array}$ & $\begin{array}{l}\text { Il est studieux, mais il aime aussi } \\
\text { s'amuser. }\end{array}$ \\
\hline \multicolumn{3}{|c|}{ FILLERS } \\
\hline 1 & $\begin{array}{l}\text { Il adore la montagne parce qu'il aime } \\
\text { bien se promener }\end{array}$ & $\begin{array}{l}\text { Il adore la montagne pasqu'il aime } \\
\text { bien se promener }\end{array}$ \\
\hline 2 & Il rentre parce qu'il est fatigué & Il rentre pasqu'il est fatigué \\
\hline 3 & $\begin{array}{l}\text { Je vais au restaurant ce soir parce que } \\
\text { c'est l'anniversaire de ma sœur }\end{array}$ & $\begin{array}{l}\text { Je vais au restaurant ce soir pasque } \\
\text { c'est l'anniversaire de ma sœur }\end{array}$ \\
\hline 4 & $\begin{array}{l}\text { J'ai pris mon parapluie parce qu'il } \\
\text { pleut }\end{array}$ & J'ai pris mon parapluie pasqu'il pleut \\
\hline 5 & $\begin{array}{l}\text { Il est allé chez le médecin hier parce } \\
\text { qu'il a mal au ventre }\end{array}$ & $\begin{array}{l}\text { Il est allé chez le médecin hier pasqu'il } \\
\text { a mal au ventre }\end{array}$ \\
\hline 6 & $\begin{array}{l}\text { Il commence à faire froid parce que } \\
\text { l'hiver approche }\end{array}$ & $\begin{array}{l}\text { Il commence à faire froid pasque } \\
\text { l'hiver approche }\end{array}$ \\
\hline 7 & $\begin{array}{l}\text { Ma mère éternue beaucoup parce } \\
\text { qu'elle est allergique au pollen }\end{array}$ & $\begin{array}{l}\text { Ma mère éternue beaucoup pasqu'elle } \\
\text { est allergique au pollen }\end{array}$ \\
\hline 8 & Tu veux aller au cinéma ce soir? & Tu veux aller au ciné ce soir? \\
\hline 9 & $\begin{array}{l}\text { Nous nous levons à sept heures parce } \\
\text { que nous avons un train à prendre }\end{array}$ & $\begin{array}{l}\text { Nous nous levons à sept heures pasque } \\
\text { nous avons un train à prendre }\end{array}$ \\
\hline 10 & $\begin{array}{l}\text { Mon père voudrait acheter cette } \\
\text { automobile }\end{array}$ & Mon père voudrait acheter cette voiture \\
\hline 11 & $\begin{array}{l}\text { Elle a arrêté le sport parce qu'elle est } \\
\text { blessée }\end{array}$ & $\begin{array}{l}\text { Elle a arrêté le sport pasqu'elle est } \\
\text { blessée }\end{array}$ \\
\hline 12 & $\begin{array}{l}\text { Tu manges très peu parce que tu n'as } \\
\text { pas souvent faim }\end{array}$ & $\begin{array}{l}\text { Tu manges très peu pasque tu n'as pas } \\
\text { souvent faim }\end{array}$ \\
\hline 13 & $\begin{array}{l}\text { Tous les jours, ma collègue fait des } \\
\text { allers-retours entre son domicile et son } \\
\text { travail }\end{array}$ & $\begin{array}{l}\text { Tous les jours, ma collègue fait des } \\
\text { allers-retours entre son domicile et son } \\
\text { boulot }\end{array}$ \\
\hline 14 & $\begin{array}{l}\text { Elle a acheté des nouvelles } \\
\text { chaussures sur Internet }\end{array}$ & $\begin{array}{l}\text { Elle a acheté des nouvelles pompes sur } \\
\text { Internet }\end{array}$ \\
\hline 15 & $\begin{array}{l}\text { Ce mois-ci, on a dépensé tout notre } \\
\text { argent }\end{array}$ & Ce mois-ci, on a dépensé tout notre fric \\
\hline 16 & Il l'a trompé avec son meilleur ami & Il l'a trompé avec son meilleur pote \\
\hline 17 & $\begin{array}{l}\text { Je travaille avec les enfants depuis } \\
\text { des années }\end{array}$ & $\begin{array}{l}\text { je travaille avec les gosses depuis des } \\
\text { années }\end{array}$ \\
\hline 18 & T'as pas trop peur? & T'as pas trop la trouille? \\
\hline 19 & $\begin{array}{l}\text { Il faut que tu lui parles, il a un gros } \\
\text { problème }\end{array}$ & $\begin{array}{l}\text { Il faut que tu lui parles, il a un gros } \\
\text { pépin }\end{array}$ \\
\hline
\end{tabular}




\begin{tabular}{|c|c|c|}
\hline 20 & Ce livre est très intéressant & Ce bouquin est très intéressant \\
\hline 21 & Comptes-tu venir ce soir? & Tu comptes venir ce soir? \\
\hline 22 & $\begin{array}{l}\text { Nous sommes allés à Paris pendant } \\
\text { les vacances }\end{array}$ & $\begin{array}{l}\text { Nous avons été à Paris pendant les } \\
\text { vacances }\end{array}$ \\
\hline 23 & $\begin{array}{l}\text { Vous voyagez souvent car vous aimez } \\
\text { voir des paysages }\end{array}$ & $\begin{array}{l}\text { Vous voyagez souvent parce que vous } \\
\text { aimez voir des paysages }\end{array}$ \\
\hline 24 & $\begin{array}{l}\text { Tu as révisé pour les partiels de } \\
\text { demain? }\end{array}$ & $\begin{array}{l}\text { T'as révisé pour les partiels de } \\
\text { demain? }\end{array}$ \\
\hline 25 & $\begin{array}{l}\begin{array}{l}\text { Ils ne savent pas qu'ils sortent } \\
\text { demain }\end{array} \\
\end{array}$ & Ils savent pas qu'ils sortent demain \\
\hline 26 & Je me demande s'il est rentré & Je me demande si il est rentré \\
\hline 27 & $\begin{array}{l}\text { On a passé une bonne soirée malgré } \\
\text { le fait que t'aies trop bu }\end{array}$ & $\begin{array}{l}\text { On a passé une bonne soirée malgré } \\
\text { que t'aies trop bu }\end{array}$ \\
\hline 28 & C'est le livre dont je parle & C'est le livre que je parle \\
\hline 29 & Il a ri à la blague que j'ai fait & Il a ri à la blague que j'ai faite \\
\hline 30 & J'ai vu le chat de ma tante & J'ai vu le chat à ma tante \\
\hline 31 & Je suis encore fâché & J'suis encore fâché \\
\hline 32 & Tu as déjà tout bu? & T'as déjà tout bu? \\
\hline 33 & $\begin{array}{l}\text { Ils ont attendu plus d'une heure chez } \\
\text { le médecin }\end{array}$ & $\begin{array}{l}\text { Ils ont attendu plus d'une heure chez le } \\
\text { métsin }\end{array}$ \\
\hline 34 & Il veut quoi, lui? & Y veut quoi, lui? \\
\hline 35 & Où c'est? & Où c'est que c'est? \\
\hline 36 & $\begin{array}{l}\text { Elle est arrivée à l'heure, entre } \\
\text { guillemets }\end{array}$ & $\begin{array}{l}\text { Elle est arrivée à l'heure, entre } \\
\text { guillmets }\end{array}$ \\
\hline 37 & Je te promets que j'essayerai demain & Je te promets que j'essaierai demain \\
\hline 38 & Elle a acheté une crème anti-âge & Elle a acheté une crème anti-yâge \\
\hline 39 & Je mangerai([e]) avec toi demain & Je mangerai([ع]) avec toi demain \\
\hline 40 & $\begin{array}{l}\text { J'ai lu Le Petit Chaperon Rouge à } \\
\text { ma sœur }\end{array}$ & $\begin{array}{l}\text { J'ai lu Le P'tit Chap'ron Rouge à ma } \\
\text { sœur }\end{array}$ \\
\hline
\end{tabular}

\footnotetext{
${ }^{\mathrm{i}}$ Le protocole PFC règle l'encodage des données sur la liaison pour la lecture à voix haute et la conversation guidée et spontanée chez des locuteurs français en différentes zones géographiques. Les fichiers audio sont sujets à une transcription qui s'intègre à la base de données.

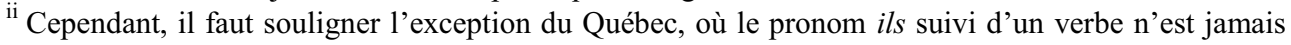
sujet à liaison, alors que la liaison dans ce contexte est considérée comme catégorique en français standard.

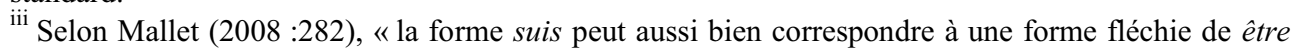
qu'à une forme de suivre. ». Elle fournit seulement les résultats bruts.
} 